\title{
Involvement of RBP4 in all-trans retinoic acid induced cleft palate
}

\author{
SHIYI DONG ${ }^{1,2}$, YADONG ZHANG ${ }^{1,2}$ and HONGZHANG HUANG ${ }^{1,2}$ \\ ${ }^{1}$ Guangdong Provincial Key Laboratory of Stomatology; ${ }^{2}$ Department of Oral and Maxillofacial Surgery, Guanghua \\ School of Stomatology, Hospital of Stomatology, Sun Yat-sen University, Guangzhou, Guangdong 510055, P.R. China
}

Received June 29, 2016; Accepted April 24, 2017

DOI: $10.3892 / \mathrm{mmr} .2017 .7327$

\begin{abstract}
The current study was designed to elucidate the mechanism of retinol binding protein 4 (RBP4) in cleft palate induced by all-trans retinoic acid (atRA). To establish a cleft palate model in $\mathrm{C} 57 \mathrm{BL} / 6 \mathrm{~J}$ mice, pregnant mice were administered atRA $(100 \mathrm{mg} / \mathrm{kg})$ by gavage at the tenth embryonic stage (E10.0). Control groups were given the equivalent volume of corn oil. Pregnant mice were dissected at E12.5, E13.5 and E14.5 to obtain the embryonic palates. The expression levels of RBP4 in the embryonic palatal mesenchyme (EPM) were determined by immunohistochemistry, reverse transcription-quantitative polymerase chain reaction (RT-qPCR) and western blotting. Human embryonic palatal mesenchymal cells were exposed to atRA to detect the variation in RBP4 induced by atRA in vitro. Small interfering RNA was used to suppress the expression of RBP4, and a plasmid overexpressing RBP4 was used to examine upregulated expression. The cell counting kit- 8 assay was used to evaluate the effect of RBP4 on cell proliferation. The expression levels of p27 and cyclin D1 were determined by RT-qPCR and western blotting, while the expression levels of extracellular signal-related kinase (ERK) $1 / 2$ and protein kinase B (AKT) were assessed by western blotting. At E14.5, RBP4 was strongly expressed in the EPM, while it was downregulated following atRA treatment, which induced cleft palate in vivo. In vitro experiments indicated that atRA suppressed the expression of RBP4 and altered the expression of p27 and cyclin D1 to cause growth inhibition. Knockdown of RBP4 resulted in decreased expression of cyclin D1 and increased p27, and suppressed proliferation. Overexpression of RBP4 reversed the inhibitory effect of atRA and promoted proliferation via the ERK $1 / 2$ and AKT signaling pathways. These results suggested that RBP4 was involved in cleft palate induced by atRA and it can be suppressed by atRA to cause growth inhibition in the embryonic palate.
\end{abstract}

Correspondence to: Dr Hongzhang Huang, Guangdong Provincial Key Laboratory of Stomatology, Sun Yat-sen University, 135 XinGangxi Road, Guangzhou, Guangdong 510055, P.R. China E-mail: huanghongzhang@yeah.net

Key words: retinol binding protein 4, cleft palate, all-trans retinoic acid, embryonic palatal mesenchyme

\section{Introduction}

Retinol binding protein 4 (RBP4) is a member of the lipocalin family and is synthesized in the liver and adipose tissue as a retinol translocator $(1,2)$. Abnormal metabolism of RBP4 can lead to disorders in the synthesis, transport and distribution of retinol, and affects embryonic growth and bone tissue differentiation (3-5). RBP4 has been demonstrated to be significantly decreased in children with non-syndromic cleft lip and/or palate (NSCLP) (6), suggesting that RBP4 levels are associated with the occurrence of NSCLP. Cleft face and palate have been observed in RBP knockout fetuses in animal models, along with obvious cranial and maxillofacial bone defects (7). These studies have indicated that RBP4 serves an important role in embryonic development, particularly in craniofacial growth. It is associated with cleft palate, in which the absence of RBP4 can lead to cleft palate.

Cleft palate resulting from multigene and environmental factors is a common malformation $(8,9)$. The formation of the secondary palate is a critical process in palatogenesis, including the growth, elevation and fusion of the palatal shelf. Failed elevation of the palatal processes is widely considered to be one of the primary causes of cleft palate and is attributed to growth inhibition of the embryonic palatal mesenchyme (EPM) (10). During the early phase of palatogenesis, large numbers of EPM cells proliferate for growth and elevation. When the proliferation of EPM cells is suppressed, the palatal processes become dysplastic and fail to elevate in an appropriate time. Palatal shelves cannot complete the contact and fusion, resulting in cleft palate.

As a derivative of vitamin A, all-trans retinoic acid (atRA) is involved in the differentiation, proliferation and apoptosis of palatogenesis (11). Previous studies have confirmed that atRA inhibits the proliferation and induces cell cycle arrest in EPM cells to cause palatal dysplasia and the development of cleft palate $(12,13)$. Previous studies have demonstrated that atRA suppresses the expression of RBP4 in adipose tissues and liver (14). However, the effect of atRA on RBP4 in the embryonic palate requires further elucidation. The present study aimed to evaluate the function of RBP4 in the development of the atRA-induced cleft palate.

\section{Materials and methods}

Animals and atRA treatment. All animal experiments were approved by the Ethics Committee for Animal Experiments 
of Sun Yat-sen University (IACUC: DB-15-0302). A total of 40 male and 40 female mice were used in the present study. The animals were housed at $22^{\circ} \mathrm{C}$ with a 12 -h light/dark cycle, and given pellet food and tap water ad libitum. Specific pathogen-free C57BL/6J male mice aged 7-8 weeks (weight, 22-25 g) and female mice aged 4-5 weeks (weight, 14-18 g) were mated. Day 0 was defined as the presence of a vaginal plug. At embryonic day 10 (E10.0), 20 pregnant mice in the experimental group were administered atRA $(100 \mathrm{mg} / \mathrm{kg}$; Sigma-Aldrich; Merck Millipore, Darmstadt, Germany) in corn oil by gavage, and 20 pregnant mice in the control group received the same volume of corn oil. The mice were euthanized on E12.5, E13.5 and E14.5 by cervical dislocation to obtain embryonic palates.

Immunohistochemistry. A total of 58 embryonic palates were fixed in $4 \%$ paraformaldehyde for $48 \mathrm{~h}$ and dehydrated using an ethanol series. Next, they were embedded in paraffin in the coronal orientation. The paraffin blocks were sliced into $4 \mu \mathrm{m}$ sections. Every section was deparaffinized and hydrated using an ethanol series. The sections were retrieved by applying citric acid buffer ( $\mathrm{pH}$ 6.0) for $30 \mathrm{~min}$ in a microwave for antigen retrieval, followed by incubation with anti-RBP4 (cat. no. ab109193; 1:200; Abcam, Cambridge, UK) for $18 \mathrm{~h}$ at $4^{\circ} \mathrm{C}$. The sections were washed with phosphate-buffered saline (PBS; Gibco; Thermo Fisher Scientific, Inc., Waltham, MA, USA) and incubated with secondary Ab-BIO (OriGene Technologies, Inc., Beijing, China) at $37^{\circ} \mathrm{C}$ for $1 \mathrm{~h}$. Staining was performed with 3,3'-diaminobenzidine (DAB; OriGene Technologies, Inc.), and the presence of brown granules was considered to indicate positive staining. Subsequently, the sections were stained with hematoxylin for $3 \mathrm{sec}$. Images were obtained using a Zeiss Axioskop 40 (Leica Microsystems $\mathrm{GmbH}$, Wetzlar, Germany).

Cell culture and treatment with atRA. Human embryonic palatal mesenchymal (HEPM) cells were purchased from the American Type Culture Collection (Manassas, VA, USA). The cells were cultured in Dulbecco's modified Eagle's medium (DMEM; Gibco, Carlsbad, CA) containing 10\% fetal bovine serum (FBS; Gibco; Thermo Fisher Scientific, Inc.) and $1 \%$ penicillin/streptomycin (Gibco; Thermo Fisher Scientific, Inc.) in a $5 \% \mathrm{CO}_{2}$ atmosphere at $37^{\circ} \mathrm{C}$. To study the effects of atRA, the cells were exposed to different concentrations of atRA $(1$ and $3 \mu \mathrm{M})$. There were 3 duplicate cultures in each group, and experiments were performed in triplicate.

Gene silencing using small interfering RNA (siRNA). HEPM cells were cultured in 6-well plates $\left(1 \times 10^{4}\right.$ cells/well $)$ in DMEM containing 10\% FBS. When the HEPM cells had reached 70\% confluency, they were transfected with a final concentration of 25 pmol RBP4 siRNA (Guangzhou RiboBio Co., Ltd., Guangzhou, China) using Lipofectamine RNAiMAX Reagent (Invitrogen; Thermo Fisher Scientific, Inc.) for $72 \mathrm{~h}$ according to the manufacturer's instructions. An irrelevant siRNA (Guangzhou RiboBio Co.,Ltd.) was used as the control. A total of 3 duplicate samples were used in each group, and experiments were performed in triplicate. These cells were collected for analysis of the associated mRNA and protein expression. Reverse transcription-quantitative polymerase chain reaction
(RT-qPCR) and western blotting were be used to verify the knockdown efficiency of the target gene.

Overexpression of RBP4. RBP4 protein was overexpressed in HEPM cells to assess the function of RBP4 in the palatal mesenchyme according to a standard transient transfection protocol. The full-length RBP4 gene was cloned into pEZ-M98-GFP (iGeneBio Biotechnology Co., Ltd., Guangzhou, China) for the experiments. Cells transfected with pEZ-M98-GFP (without RBP4) were used as the control group. The HEPM cells were seeded into 6-well plates (1x10 cells/well) in DMEM containing 10\% FBS. When the HEPM cells had reached 70\% confluency, they were transfected with the above-mentioned constructs in Opti-MEM medium (Gibco; Thermo Fisher Scientific, Inc.), and Lipofectamine 3000 reagent (Invitrogen; Thermo Fisher Scientific, Inc.) was used for transfecting nucleic acids ( $2500 \mathrm{ng} /$ well) into cells according to standard procedures. Following transfection for $48 \mathrm{~h}$, the medium was refreshed, and the cells were exposed to atRA $(3 \mu \mathrm{M})$ for $48 \mathrm{~h}$. A total of 3 duplicate samples were used in each group, and experiments were performed in triplicate. The efficiency of the target gene overexpression was evaluated by western blotting.

$R T-q P C R$. Total RNA was extracted from mouse embryonic palates (60 in each group) or HEPM cells using a Qiagen RNeasy Mini kit (Qiagen GmbH, Hilden, Germany) according to the instructions provided by the manufacturer. A total of $\sim 20 \mu \mathrm{l}$ cDNA was synthesized from $1 \mu \mathrm{g}$ of total RNA in a $20 \mu 1$ reaction mixture. The reaction mixture included total RNA, Anchored-oligo (dT)18 Primer, Random Hexamer Primer, PCR-grade $\mathrm{H}_{2} \mathrm{O}$, Transcriptor RT Reaction Buffer (5X), Protector RNase Inhibitor, Deoxynucleotide Mix and Transcriptor Reverse Transcriptase (all Roche Diagnostics $\mathrm{GmbH}$, Mannheim, Germany). RT-qPCR was conducted using the Light Cycler 480 System (Roche Diagnostics $\mathrm{GmbH}$ ) with SYBR Green I Master Mix (Roche Diagnostics GmbH) to quantitatively measure the mRNA levels. $\beta$-actin and GAPDH were used as reference genes. The following PCR primers (Generay Biotech Co., Ltd., Shanghai, China) were used in the experiments: RBP4 (Mus), forward 5'-GACACGGACTAC GACACC-3' and reverse 5'-CACGAGAAAACACAAAGG A-3'; $\beta$-actin (Mus), forward 5'-TCACCCACACTGTGCCCA TCTACGA-3' and reverse 5'-GGATGCCACAGGATTCCA TACCCA-3'; RBP4 (homo), forward 5'-GAGGACCCTGCC AAGTTCA-3' and reverse 5'-GGGAAAACACGAAGGAGT AGC-3'; P27 (homo), forward 5'-CAAACGTGCGAGTGT CTA-3' and reverse 5'-CAGTGCTTCTCCAAGTCC-3'; cyclin D1 (homo), forward 5'-TCCTACTACCGCCTCACA-3' and reverse 5'-ACCTCCTCCTCCTCCTCT-3'; GAPDH (homo), forward 5'-GGACCTGACCTGCCGTCTAG-3' and reverse 5'-GTAGCCCAGGATGCCCTTGA-3'.

Western blot analysis. 60 mouse embryonic palates in each group were treated with $1 \mathrm{U} / \mathrm{ml}$ dispase II (Roche Diagnostics, Indianapolis, IN, USA) for $15 \mathrm{~min}$ at $37^{\circ} \mathrm{C}$ to remove epithelia and obtain EPM at E12.5, E13.5 and E14.5. The palatal mesenchyme was flushed with cold PBS three times and lysed in cell lysis buffer (10\% RIPA, $1 \%$ protease inhibitor, $1 \%$ phosphatase inhibitor; Sigma-Aldrich; Merck Millipore) on ice for $30 \mathrm{~min}$. The protein was collected after the lysate 
A
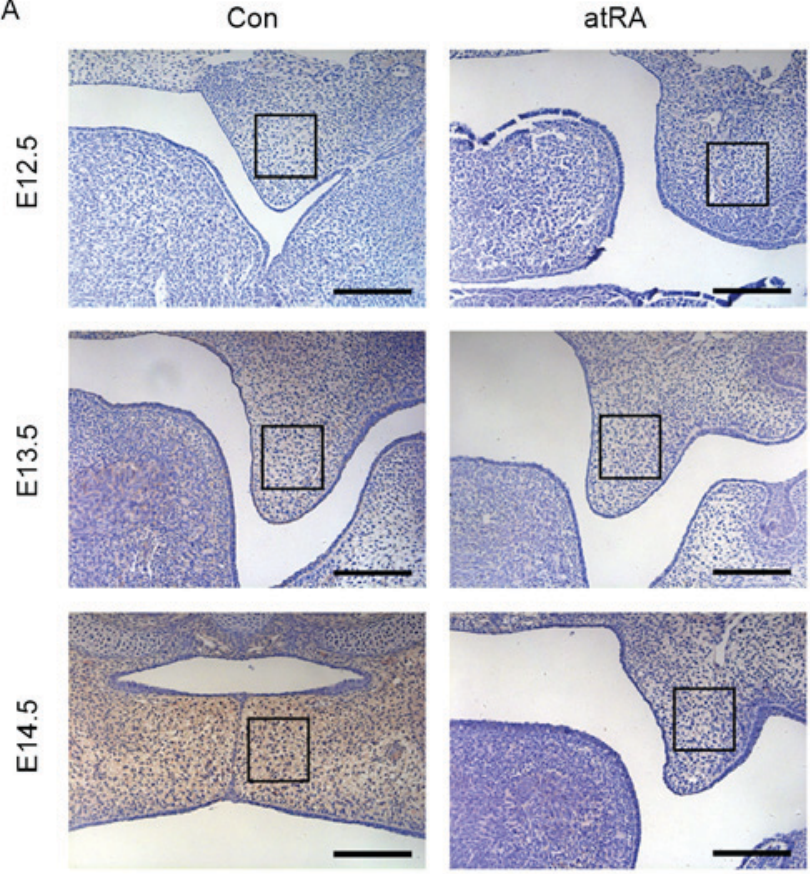

B

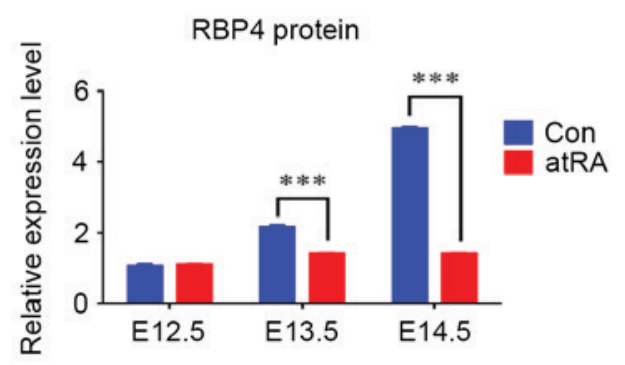

Figure 1. Immunohistochemistry assay demonstrating RBP4 expression in the EPM. RBP4 was significantly decreased at E13.5 and E14.5 in the EPM exposed to atRA when compared with the Con group (A) Immunohistochemistry results (scale bar, $50 \mu \mathrm{m}$ ), (B) quantification. ${ }^{* * *} \mathrm{P}<0.001$. RBP4, retinol binding protein 4; EPM, embryonic palatal mesenchyme; E, embryonic day; atRA, all-trans retinoic acid; Con, control.

solution was centrifuged at $4^{\circ} \mathrm{C}$ and $14,000 \times \mathrm{g}$ for $30 \mathrm{~min}$. The protein $(30 \mu \mathrm{g})$ was separated by $10 \%$ SDS-PAGE (CWBIO, Beijing, China) and transferred onto polyvinylidene difluoride membranes. The membrane was blocked in $5 \%$ bovine serum albumin (Beyotime Institute of Biotechnology, Shanghai, China) for $1 \mathrm{~h}$ and incubated overnight at $4^{\circ} \mathrm{C}$ with the following antibodies: Rabbit monoclonal anti-RBP4 (cat. no. ab109193; 1:1,000; Abcam), rabbit monoclonal anti-p27 (cat. no. 3688s; 1:1,000; Cell Signaling Technology, Inc., Danvers, MA, USA) and rabbit monoclonal anti-cyclin D1 (cat. no. ab134175; 1:2,000; Abcam) antibodies. The membrane was incubated with horseradish peroxidase-coupled secondary antibodies for $1 \mathrm{~h}$ following three washes with Tris-buffered saline/Tween 20 (CWBIO). The membrane was detected using chemiluminescence with Immobilon western chemiluminescent horseradish-peroxidase substrate (EMD Millipore, Billerica, MA, USA).

Protein derived from cultured HEPM cells was also detected by western blotting. The following steps were repeated as described previously (15). In addition to the antibodies mentioned above, the following antibodies were used: Rabbit monoclonal anti-extracellular signal-related kinase (ERK) 1/2 (cat. no. 4695s; 1:1,000; Cell Signaling Technology, Inc.), rabbit monoclonal anti-phosphorylated (p)-ERK1/2 (cat. no. 4370s; Thr202/Tyr204; 1:2,000; Cell Signaling Technology, Inc.), rabbit monoclonal anti-AKT (cat. no. 4691s; 1:1,000; Cell Signaling Technology, Inc.), rabbit monoclonal anti-p-AKT (cat. no. 4060s; Ser473, 1:1,000; Cell Signaling Technology, Inc.), rabbit monoclonal anti-proliferating cell nuclear antigen (PCNA) (cat. no. 13110s; 1:1,000; Cell Signaling Technology, Inc.).

Cell counting kit-8 (CCK8) assay. HEPM cells were cultured in a 96 -well plate $\left(5,000\right.$ cells/well) in DMEM at $37^{\circ} \mathrm{C}$ for $24 \mathrm{~h}$. The medium was then replaced with fresh DMEM, and the cultures were exposed to atRA $(3 \mu \mathrm{M})$ or the transfection complex. Following 24, 48, 72 and 96 h, $10 \mu \mathrm{l} \mathrm{CCK8} \mathrm{(Dojindo}$ Molecular Technologies, Inc., Tokyo, Japan) was added to each well for $2 \mathrm{~h}$ at $37^{\circ} \mathrm{C}$. The absorbance was measured using a microplate reader at $450 \mathrm{~nm}$.

Statistical analysis. All investigations, including individual experiments, were performed in triplicate. All data were analyzed using SPSS software, version 13.0 (SPSS, Inc., Chicago, IL, USA) with the Student's t-test, or one way analysis of variance followed by the Bonferroni post hoc test to correct for multiple comparisons. The results are presented as the mean values \pm standard deviation, and $\mathrm{P}<0.05$ was considered to indicate a statistically significant difference.

\section{Results}

RBP4 was downregulated in the EPM of cleft palate induced by atRA. At E12.5, the palate grew vertically along the side of the tongue, and the expression of RBP4 was negative without apparent differences in the EPM in either group (Fig. 1). At E13.5, the palatal shelves still grew in a vertical position alongside the tongue, and they appeared smaller in the atRA-treated group than that in the control group (Fig. 1A). There were more RBP4-positive cells in the EPM in the control group compared with the atRA group (Fig. 1; $\mathrm{P}<0.001)$. At E14.5, the bilateral palates shifted toward a horizontal position to contact one another in the control group. During this period, the medial edge epithelium was contacted to develop into the medial edge epithelial seam, and finally palate shelves fused. The expression of RBP4 was strongly positive in the EPM (Fig. 1A). By contrast, the palatal shelves appeared abnormally small and failed to undergo elevation and fusion, finally developing into cleft palate in the atRA-treated group at E14.5 (Fig. 1A). The incidence of cleft palate in the atRA-exposed groups was $96.3 \%$ (78 of 81 embryos). RBP4 was significantly downregulated in the EPM in atRA treatment compared with the control group (Fig. 1; P<0.001). The RT-qPCR results indicated that the mRNA levels of RBP4 were reduced at E13.5 $(\mathrm{P}<0.05)$ and downregulated at E14.5 in the atRA compared with the control group (Fig. 2A; $\mathrm{P}<0.01$ ). Western blotting results identified that from E12.5 to E14.5, the protein levels of RBP4 were significantly reduced in the atRA-exposed vs. the control EPM, particularly at E14.5 (Fig. 2B; P<0.001). 
A

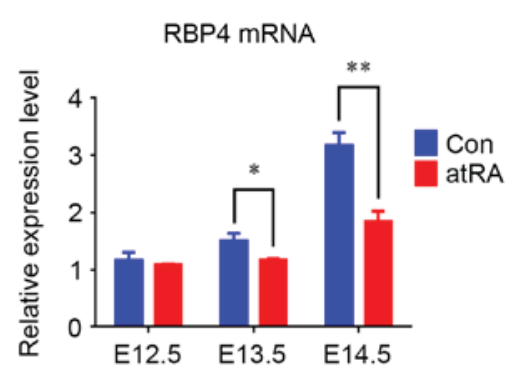

B

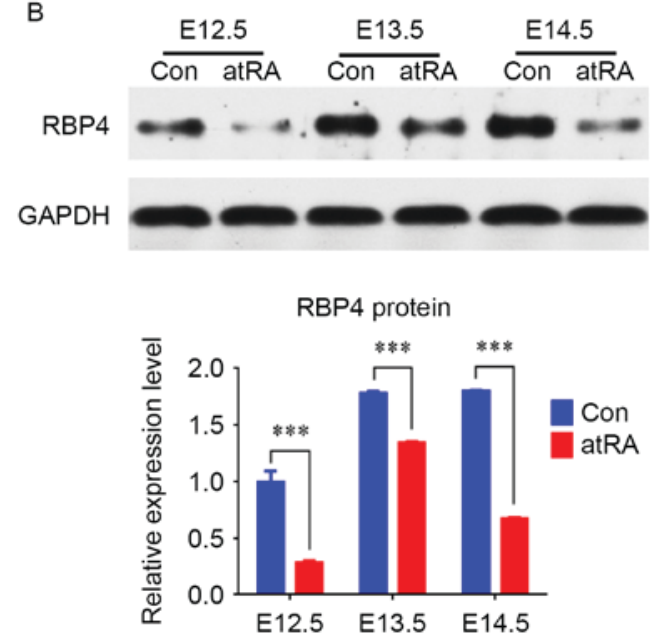

Figure 2. The expression of RBP4 was downregulated in the EPM treated with atRA. (A) When compared with the Con group, mRNA expression of RBP4 was reduced in the atRA group at E13.5 and E14.5. (B) Protein expression of RBP4 from E12.5 to E14.5 was lower in the atRA groups vs. Con. ${ }^{*} \mathrm{P}<0.05,{ }^{* *} \mathrm{P}<0.01$ and ${ }^{* * *} \mathrm{P}<0.001$. RBP4, retinol binding protein 4 ; EPM, embryonic palatal mesenchyme; atRA, all-trans retinoic acid; E, embryonic day; Con, control.

atRA induced RBP4 downregulation and regulated the expression of p27, cyclin D1, PCNA and the associated signaling pathway. To evaluate the effect of atRA on RBP4, HEPM cells were exposed to different concentrations of atRA (1 and $3 \mu \mathrm{M}$ ). The results indicated that the mRNA levels of RBP4 were decreased in HEPM cells treated with atRA (1 and $3 \mu \mathrm{M}$ ) (Fig. 3A; $<<0.01$ ). The protein levels of RBP4 were also decreased in the atRA-treated groups compared with the controls (Fig. 4; $\mathrm{P}<0.001$ ). In contrast, elevated p27 mRNA levels (Fig. 3B; $\mathrm{P}<0.05$ ) and protein levels (Fig. 4; $\mathrm{P}<0.001$ ) were observed in HEPM cells exposed to atRA ( 1 and $3 \mu \mathrm{M})$. Cyclin D1 mRNA and protein levels were suppressed in the atRA-exposed groups compared with the control group (Figs. $3 \mathrm{C}$ and 4 ; $\mathrm{P}<0.001$ ). The protein levels of PCNA were reduced in the atRA-exposed groups compared with the controls (Fig. 4). Western blotting results identified that p-ERK1/2 was reduced in atRA-treated cells (Fig. 4; P<0.01), and $\mathrm{p}-\mathrm{AKT}$ was also reduced compared with the control group (Fig. 4; $\mathrm{P}<0.001)$.

RBP4 suppressed the expression of p27 and stimulated the expression of cyclin D1 via the AKT and ERK1/2 signaling pathway. siRNA knockdown of RBP4 was performed to evaluate its involvement in cell proliferation (Figs. 5 and 6). The RT-qPCR and western blotting results demonstrated that
A

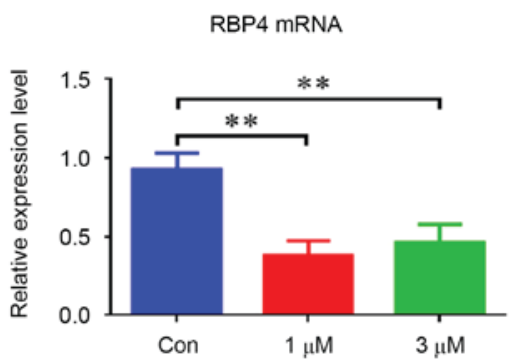

B

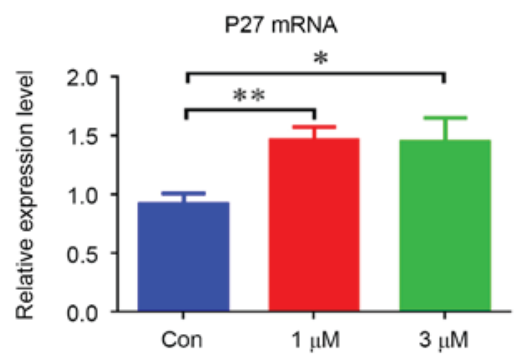

C

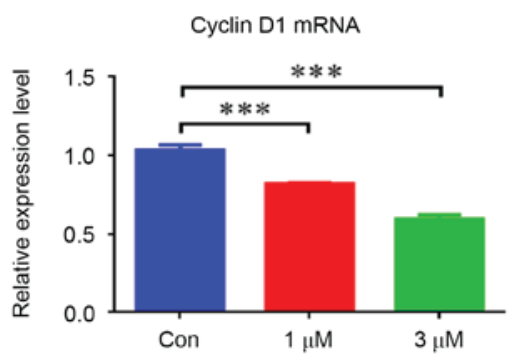

Figure 3. atRA suppressed RBP4 mRNA levels and altered the mRNA levels of p27 and cyclin D1 in human embryonic palatal mesenchymal cells. (A) mRNA expression of RBP4 was decreased in the atRA-treated group compared with that in the Con group. (B) mRNA expression of p27 was increased in the atRA-treated group compared with the Con group. (C) mRNA expression of cyclin D1 was reduced in the atRA-treated group compared with the Con group. There were 3 duplicate cultures in each group, and experiments were performed in triplicate. ${ }^{*} \mathrm{P}<0.05,{ }^{* *} \mathrm{P}<0.01$ and ${ }^{* * *} \mathrm{P}<0.001$. atRA, all-trans retinoic acid; RBP4, retinol binding protein 4; Con, control.

RBP4 was effectively knocked down by siRNA both at the mRNA and protein levels; mRNA levels were repressed by $84.1 \%$ ( $0.17 \pm 0.06$ vs. $1.07 \pm 0.12 ; \mathrm{P}<0.001$; Fig. $5 \mathrm{~A})$, and protein levels were repressed by $56.7 \%(0.45 \pm 0.02$ vs. $1.04 \pm 0.05$; $\mathrm{P}<0.001$ ) (Fig. 6). Downregulation of RBP4 in HEPM cells transfected with RBP4 siRNA resulted in decreased expression of cyclin D1 at both mRNA (Fig. 5C; $\mathrm{P}<0.01$ ) and protein (Fig. 6) levels $(\mathrm{P}<0.001)$, while increased p27 mRNA (Fig. 5B; $\mathrm{P}<0.05$ ) and protein (Fig. 6) levels were observed $(\mathrm{P}<0.001)$. The protein levels of PCNA were reduced in RBP4-siRNA HEPM cells compared with the control group (Fig. 6; $\mathrm{P}<0.01$ ). The western blotting results demonstrated that $\mathrm{p}$-ERK1/2 and $\mathrm{p}-\mathrm{AKT}$ were reduced along with RBP4 (Fig. 6; $\mathrm{P}<0.001$ ). The western blotting results indicated that RBP4 was effectively upregulated in response to RBP4 overexpression in HEPM cells and the degree of overexpression was $51.2 \%$ ( $1.50 \pm 0.02$ vs. $0.99 \pm 0.01 ; \mathrm{P}<0.001)$, cyclin D1 was also upregulated $(\mathrm{P}<0.001)$, while p27 was downregulated (Fig. 7; $\mathrm{P}<0.001$ ). In the atRA-exposed group, RBP4 levels and cyclin D1 were decreased (Fig. 7; P<0.01), however p27 was increased (Fig. 7; $\mathrm{P}<0.001$ ), as compared with the control 

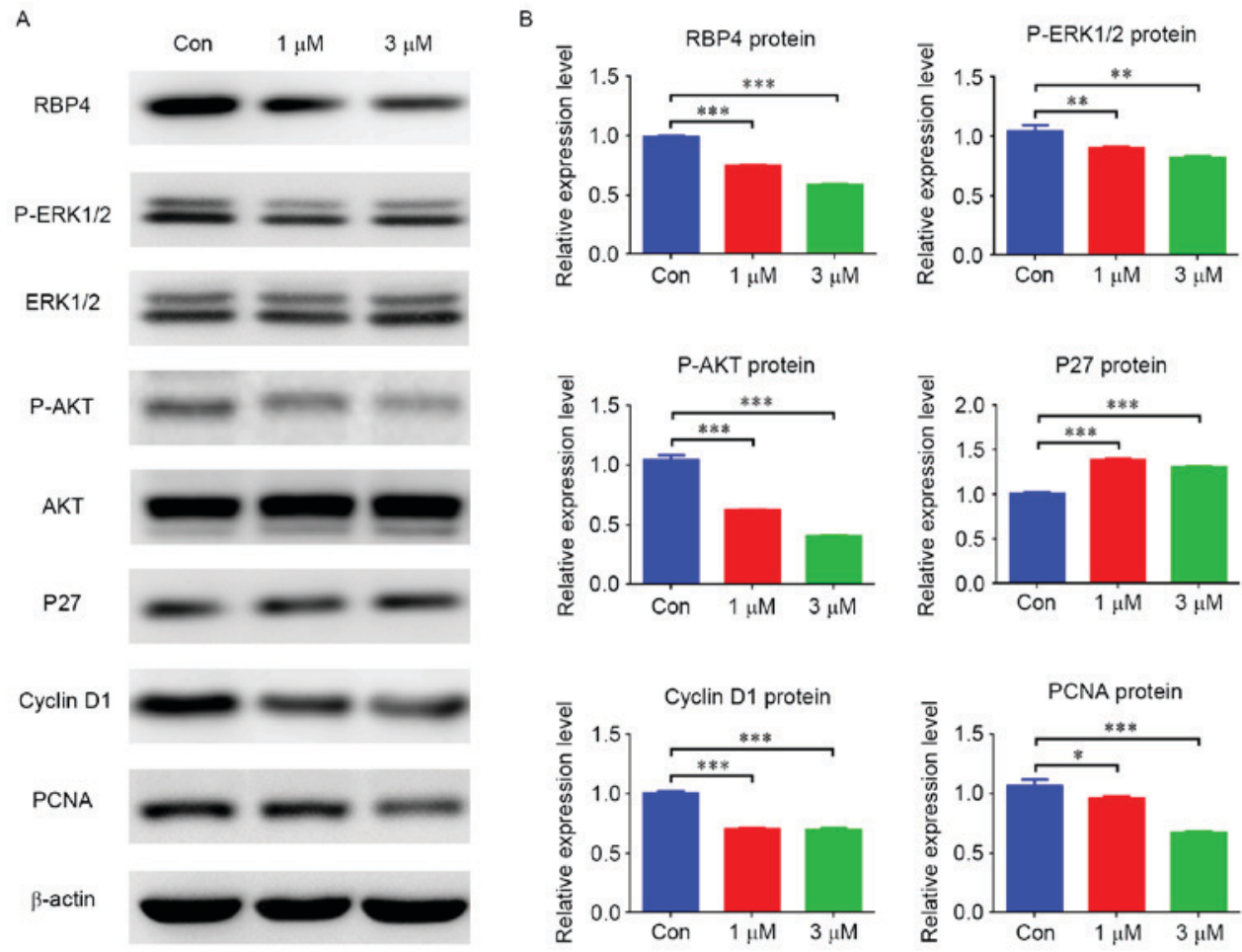

Figure 4. atRA inhibited RBP4 protein levels and altered the expression of associated regulators and signaling pathways in human embryonic palatal mesenchymal cells. (A) Protein levels of RBP4, cyclin D1, PCNA, p-ERK1/2 and p-AKT were all decreased in the atRA-treated group, however p27 was increased compared with the Con group. (B) Data for the protein levels are presented as the mean + standard deviation. There were 3 duplicate cultures in each group, and experiments were performed in triplicate. ${ }^{*} \mathrm{P}<0.05,{ }^{* *} \mathrm{P}<0.01$ and ${ }^{* * *} \mathrm{P}<0.001$. atRA, all-trans retinoic acid; RBP4, retinol binding protein 4 ; PCNA, proliferating cell nuclear antigen; p-, phosphorylated; ERK, extracellular signal-related kinase; AKT, protein kinase B; Con, control.

group. By contrast, RBP4 and cyclin D1 were not downregulated (Fig. 7; P<0.001) and p27 levels did not increase (Fig. 7; $\mathrm{P}<0.001)$ in the RBP4 overexpression plus atRA-treated group compared with the control. The western blot results additionally indicated that p-ERK $1 / 2$ and p-AKT were both upregulated in response to RBP4 overexpression in HEPM cells (Fig. 7; $\mathrm{P}<0.001$ ), and they were not downregulated in the RBP4 overexpression plus atRA-treated group $(\mathrm{P}<0.001)$ compared with the control.

RBP4 promoted the proliferation of HEPM cells by CCK8 assay. When HEPM cells were exposed to atRA (3 $\mu \mathrm{M})$, cell proliferation levels were reduced from 24 to $96 \mathrm{~h}$ (Fig. 8A). siRNA knockdown of RBP4 in HEPM cells resulted in markedly reduced cell proliferation levels from 48 to $96 \mathrm{~h}$ (Fig. 8B). By contrast, RBP4 overexpression in HEPM cells resulted in enhanced cell proliferation levels from 48 to $96 \mathrm{~h}$, with maximum proliferation observed at $96 \mathrm{~h}$ (Fig. 8C; $\mathrm{P}<0.01$ ). Compared with the control group, when the RBP4-overexpressing HEPM cells were exposed to atRA, cell proliferation levels were maintained, particularly at $72 \mathrm{~h}$ (Fig. 8C; $\mathrm{P}<0.001)$.

\section{Discussion}

atRA is essential for the development of embryos, by regulating morphogenesis, cell proliferation and differentiation $(11,16)$. Embryos develop normally under low concentrations of atRA, while when exposed to overdoses of atRA, they develop defects in both animals and humans, such as cleft palate $(16,17)$. atRA has been widely used as a teratogen to establish animal models and cytotoxicity tests to determine the pathogenesis of cleft palate. Previous studies have confirmed that in pregnant mice exposed to atRA $(100 \mathrm{mg} / \mathrm{kg})$ at E10.0, the palatal shelves become dysplastic and fail to contact one another, resulting in cleft palate $(17,18)$. In the mouse, palate shelves initially grow vertically alongside the tongue at E12.5 and then elevate to the horizontal position above the tongue, and contact with each other and fuse at E14.5 (19,13). Normal growth of EPM serves a key role in this process, a previous study indicated that atRA suppressed the process of growth and differentiation in the developing mesenchyme to cause an abnormally small palate and unfused palatal shelves (20). atRA has been demonstrated to inhibit the proliferation of EPM cells to cause cleft palate (13). The results additionally indicated that the palatal shelves were abnormally small and dysplastic in the atRA-exposed embryos, and unable to shift horizontally due to the inhibition effect of atRA.

$\mathrm{RBP} 4$ is a $21 \mathrm{kDa}$ protein that is located in 10q23-24 in humans (21). RBP4 is primarily synthesized in the liver and transported to target tissues; it can also be detected in extrahepatic tissues such as adipose tissue, the kidneys and the lungs (22). RBP4, which is previously known as RBP, participates in retinol translocation, lipid metabolism, fetal growth, bone growth and inflammatory responses $(23,24)$. A study reported that RBP knockout mouse have morphological abnormalities, including cleft palate and maxillofacial bone defects (7). This indicated that RBP4 serves an important role in normal physiological function and development, 
A

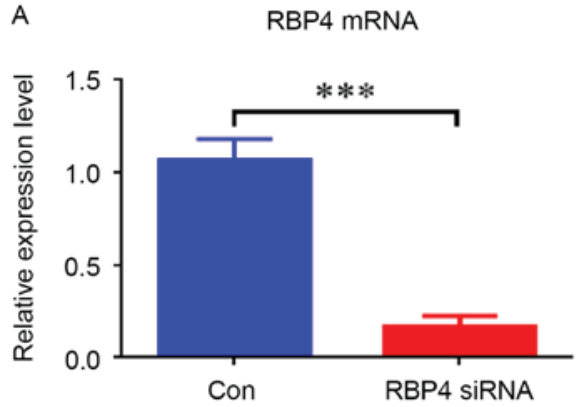

B

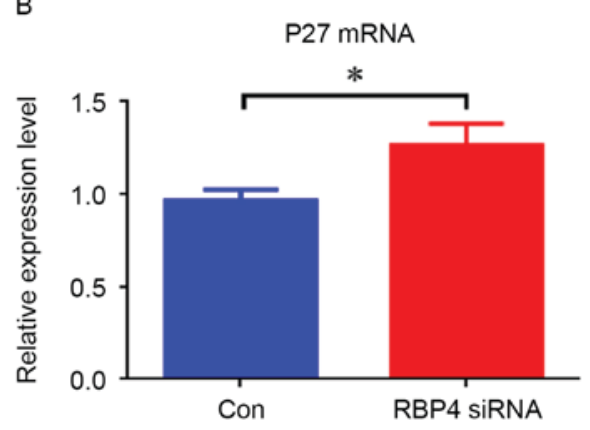

C

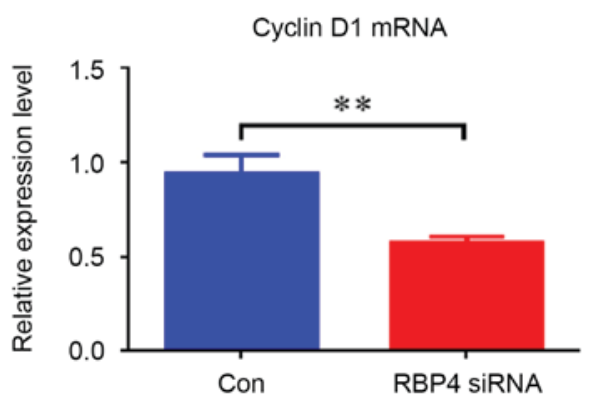

Figure 5. Knockdown of RBP4 induced upregulation of p27 and downregulation of cyclin D1 at the mRNA level. (A) When compared with the Con group, RBP4 mRNA levels were downregulated in HEPM cells treated with RBP4 siRNA. (B) P27 mRNA levels were upregulated in HEPM cells treated with RBP4 siRNA via Con. (C) Cyclin D1 mRNA levels were downregulated in HEPM cells treated with RBP4 siRNA compared with Con. A total of 3 duplicate samples were used in each group, and experiments were performed in triplicate. ${ }^{*} \mathrm{P}<0.05,{ }^{* *} \mathrm{P}<0.01$ and ${ }^{* * *} \mathrm{P}<0.001$. RBP4, retinol binding protein 4; HEPM, human embryonic palatal mesenchymal; siRNA, small interfering RNA; Con, control siRNA.

particularly in that of the palate and the maxillofacial region. It has been observed that the level of serum RBP4 is significantly lower in children with NSCLP than in those without (6). However, whether RBP4 can be detected in the EPM and the mechanism of RBP4 in cleft palate remains unclear. The present study observed that RBP4 was strongly expressed in the EPM at E13.5 and E14.5 in the normal embryonic palate, while it was downregulated in cleft palate induced by atRA. These observations indicated that RBP4 was involved in the development of the EPM and that the deficiency in RBP4 was associated with the occurrence of cleft palate.

In order to elucidate the mechanism of RBP4 in the cleft palate induced by atRA, HEPM cells were used in the in vitro experiments. The levels of RBP4 mRNA and protein were downregulated by atRA in HEPM cells. This observation was consistent with the results of the in vivo animal experiments, demonstrating that atRA can significantly inhibit the expression of RBP4. Numerous studies have demonstrated that atRA can suppress the proliferation via the upregulation of p21/p27 and the downregulation of cyclin D1 in various cell lines (25-27). P27 structurally homologous to p21 is known as cyclin-dependent kinase inhibitor, which serves a negative role in cell proliferation and cell cycle progression $(28,29)$. p27 expression can cause cell cycle arrest, while downregulation of p27 leads to cell cycle progression and cell proliferation $(28,29)$. Cyclin D1 belongs to the highly conserved cyclin family, and function as regulator of cyclin-dependent protein kinases, promoting cell cycle progression (30). PCNA, a cofactor of DNA polymerase, is widely used as a proliferation associated marker (31). The results indicated that the expression of p27 was increased and cyclin D1 and PCNA were decreased in HEPM cells exposed to atRA, indicating that atRA inhibited the proliferation of HEPM cells. The CCK8 assay also proved that atRA had an inhibitory effect on cell proliferation. The western blotting results also indicated that p-AKT and p-ERK1/2 were both reduced in HEPM cells treated with atRA. It has been reported that the AKT and ERK1/2 signaling pathways are involved in proliferation and survival in different cells (32). Activation of AKT signaling promotes DNA synthesis and cell cycle progression, regulating cellular proliferation and survival (28). ERK1/2 has been demonstrated to be an essential signaling pathway to modulate cell proliferation and apoptosis (33). The results of the present study indicated that they may be involved in cell proliferation in HEPM cells.

Previous reports have confirmed that RBP4 can enhance proliferation in vascular smooth muscle cells and rat aortic smooth muscle cells induced by hyperinsulinism $(34,35)$. However, the effect of RBP4 on HEPM cells remains unclear. In the current study, the CCK8 assay demonstrated that RBP4 enhanced the proliferation of HEPM cells, and the growth inhibition induced by atRA was mitigated by RBP4 overexpression. RBP4 has been previously observed to promote cell proliferation by upregulating p-ERK $1 / 2$ in rat aortic smooth muscle cells (35). A previous study additionally confirmed that RBP4 can significantly increase the expression of p-AKT in vascular endothelial cells (36). In the present study, results suggested that RBP4 could upregulate the expression of p-ERK1/2 and p-AKT to activate the AKT and ERK1/2 signaling pathways in HEPM cells. However, the mechanism of how RBP4 affects p-ERK1/2 and p-AKT remains unclear, and further research is required. A previous study observed that activation of the ERK1/2 and AKT signaling pathways suppresses the expression of cyclin-dependent kinase inhibitors (p27 and p21) and increases the expression of cell cyclins (cyclin D1 and cyclin E) to promote cell cycle progression and cell proliferation (32). The results of the present study demonstrated that RBP4 could stimulate cyclin D1 and inhibit p27 to promote cell proliferation via the AKT and ERK1/2 signaling pathways. When RBP4 was suppressed, it can cause growth inhibition. The in vivo and in vitro studies indicated that depression of RBP4 in the palate could suppress the expression of p-ERK $1 / 2$ and p-AKT, and affect the expression of p27 and cyclin D1 to cause growth inhibition of EPM, leading to cleft palate. The results demonstrated that RBP4 served an 
A

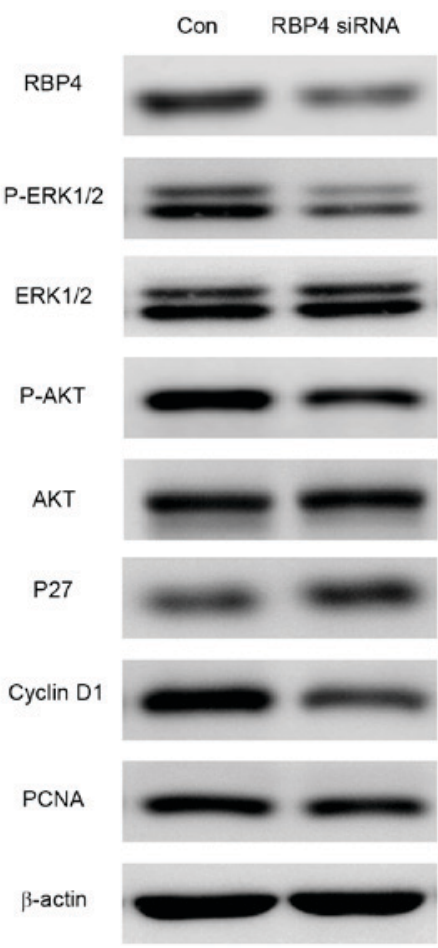

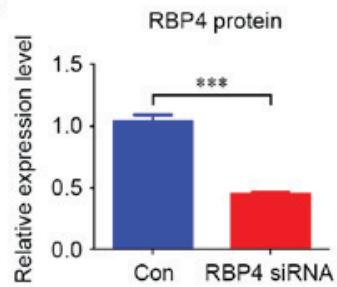

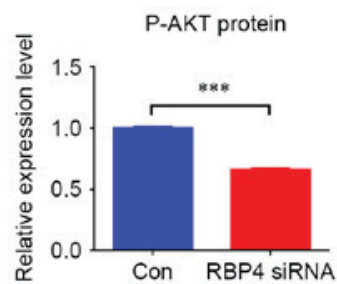

Cyclin D1 protein

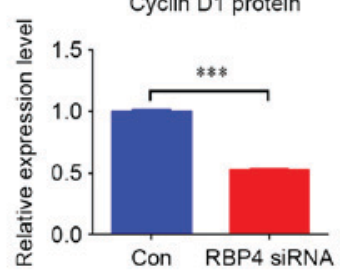

P-ERK1/2 protein
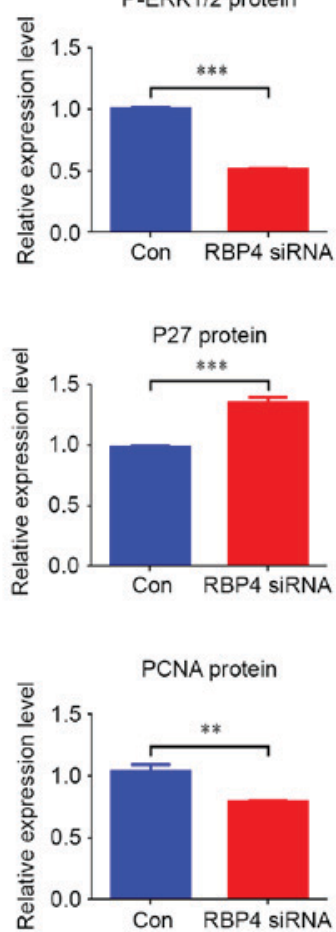

Figure 6. Knockdown of RBP4 altered the expression of related regulators and signaling pathways in HEPM cells. (A) Protein levels of RBP4, cyclin D1, PCNA, p-ERK1/2 and p-AKT were all downregulated in HEPM cells treated with RBP4 siRNA, however p27 was upregulated compared with the Con group. (B) Data for the protein levels are presented as the mean + standard deviation. A total of 3 duplicate samples were used in each group, and experiments were performed in triplicate. ${ }^{* *} \mathrm{P}<0.01$ and ${ }^{* * *} \mathrm{P}<0.001$. RBP4, retinol binding protein 4; HEPM, human embryonic palatal mesenchymal; PCNA, proliferating cell nuclear antigen; p-, phosphorylated; ERK, extracellular signal-related kinase; AKT, protein kinase B; siRNA, small interfering RNA; Con, control siRNA.

A

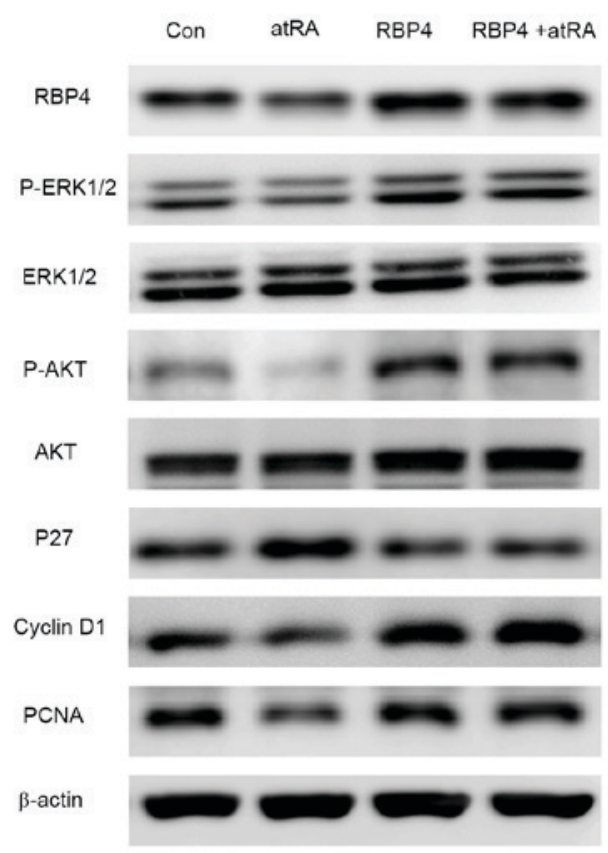

B
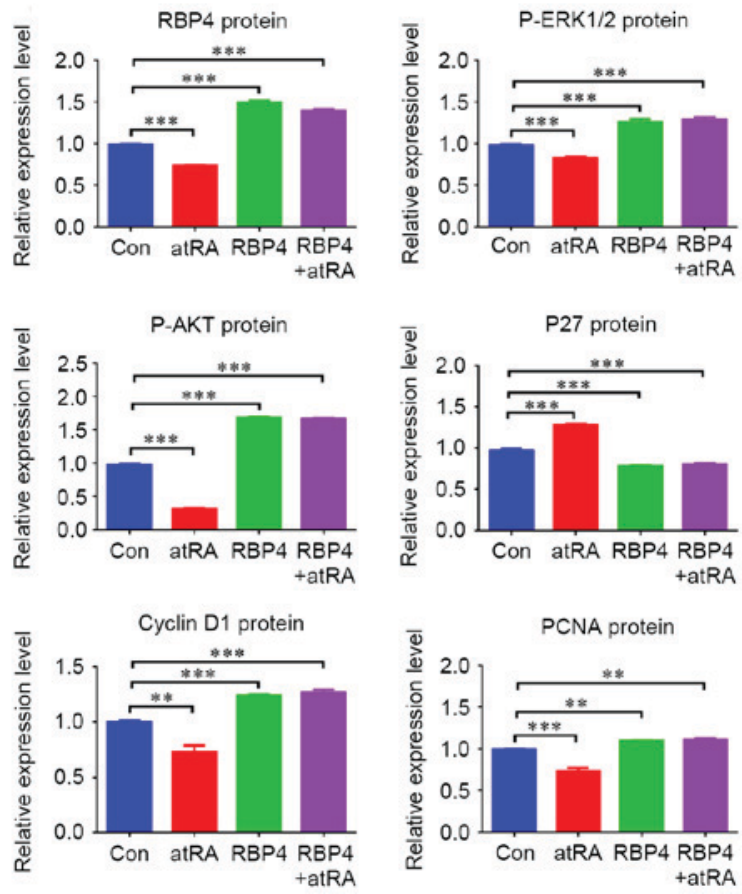

Figure 7. Overexpression of RBP4 altered the expression of related regulators and related signal pathways in HEPM cells. (A) The protein levels of RBP4, cyclin D1, PCNA, p-ERK1/2 and p-AKT were all upregulated in HEPM cells overexpressing RBP4, however p27 was downregulated compared with the Con group. The protein levels of RBP4, cyclin D1, PCNA, p-ERK1/2 and p-AKT remained upregulated in the RBP4 overexpressing group treated with atRA, and p27 was downregulated compared with the Con group. (B) The data for the protein levels are presented as the mean + standard deviation. A total of 3 duplicate samples were used in each group, and experiments were performed in triplicate. ${ }^{* *} \mathrm{P}<0.01$ and ${ }^{* * *} \mathrm{P}<0.001$. RBP4, retinol binding protein 4 ; HEPM, human embryonic palatal mesenchymal; PCNA, proliferating cell nuclear antigen; p-, phosphorylated; ERK, extracellular signal-related kinase; AKT, protein kinase $\mathrm{B}$; atRA, all-trans retinoic acid; Con, control. 


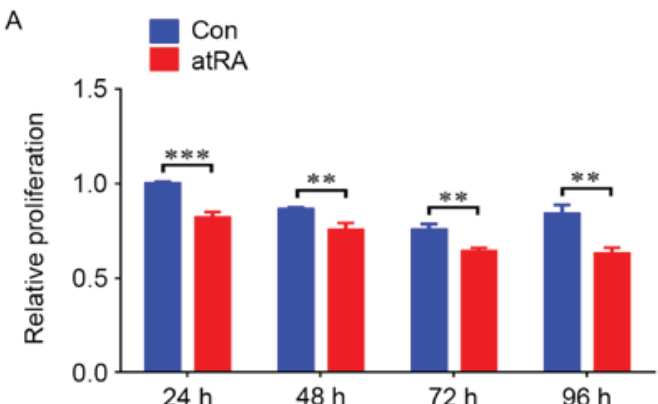

B

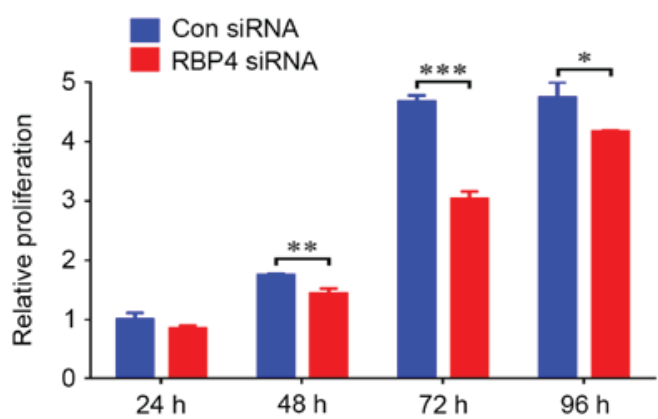

C

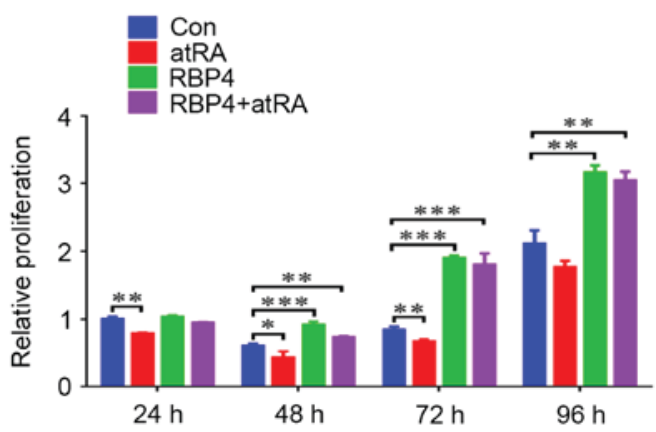

Figure 8. Effects of RBP4 on HEPM cells using the cell counting kit-8 assay. (A) The proliferation levels of HEPM cells treated with atRA $(3 \mu \mathrm{M})$ decreased from 24-96 h. (B) The proliferation levels of HEPM cells transfected with RBP4 siRNA decreased from 48-96 h. (C) The proliferation levels of HEPM cells overexpressing RBP4 increased from 24-96 h, and atRA had no effect on these cells. A total of 3 duplicate samples were used in each group, and experiments were performed in triplicate. ${ }^{*} \mathrm{P}<0.05,{ }^{* *} \mathrm{P}<0.01$ and ${ }^{* * *} \mathrm{P}<0.001$. RBP4, retinol binding protein 4; HEPM, human embryonic palatal mesenchymal; siRNA, small interfering RNA; atRA, all-trans retinoic acid; Con, control.

important role in cleft palate induced by atRA, indicating that it could be used as detection index or prophylaxis method for cleft palate.

In summary, the study confirmed that RBP4 is involved in cleft palate induced by atRA, and it is downregulated in the EPM treated with atRA. RBP4 can be suppressed by atRA to cause growth inhibition in the embryonic palate.

\section{Acknowledgements}

The current study was funded by grants from the National Natural Science Foundation of China (grant no. 81100739), the Project of Health and Family Planning Commission of Shenzhen Municipality (grant no. 201302202) and the Natural Science Foundation of Guangdong Province (grant no. S2011040004190).

\section{References}

1. Noy N: Retinoid-binding proteins: Mediators of retinoid action. Biochem J 348: 481-495, 2000.

2. Yang Q, Graham TE, Mody N, Preitner F, Peroni OD, Zabolotny JM, Kotani K, Quadro L and Kahn BB: Serum retinol binding protein 4 contributes to insulin resistance in obesity and type 2 diabetes. Nature 436: 356-362, 2005.

3. Munkhtulga L, Nakayama K, Utsumi N, Yanagisawa Y, Gotoh T, Omi T, Kumada M, Erdenebulgan B, Zolzaya K, Lkhagvasuren T and Iwamoto S: Identification of a regulatory SNP in the retinol binding protein 4 gene associated with type 2 diabetes in Mongolia. Hum Genet 120: 879-888, 2007.

4. Chan TF, Tsai YC, Wu CH, Lee CH, Wang SH and Su JH: The positive correlation between cord serum retinol-binding protein 4 concentrations and fetal growth. Gynecol Obstet Invest 72: 98-1028, 2011.

5. Hatfield JT, Anderson PJ and Powell BC: Retinol-binding protein 4 is expressed in chondrocytes of developing mouse long bones: Implications for a local role in formation of the secondary ossification center. Histochem Cell Biol 139: 727-734, 2013.

6. Zhang J, Zhou S, Zhang Q, Feng S, Chen Y, Zheng H, Wang X, Zhao W, Zhang T, Zhou Y, et al: Proteomic analysis of RBP4/Vitamin A in children with cleft lip and/or palate. J Dent Res 93: 547-552, 2014.

7. Quadro L, Hamberger L, Gottesman ME, Wang F, Colantuoni V, Blaner WS and Mendelsohn CL: Pathways of vitamin A delivery to the embryo: Insights from a new tunable model of embryonic vitamin A deficiency. Endocrinology 146: 4479-4490, 2005

8. Marazita ML and Mooney MP: Current concepts in the embryology and genetics of cleft lip and cleft palate. Clin Plast Surg 31: 125-140, 2004.

9. Wyszynski DF and Beaty TH: Phenotypic discordance in a family with monozygotic twins and nonsyndromic cleft lip and palate: Follow-up. Am J Med Genet 110: 182-183, 2002.

10. Murray JC and Schutte BC: Cleft palate: Players, pathways, and pursuits. J Clin Invest 113: 1676-1678, 2004.

11. Clagett-Dame $M$ and DeLuca HF: The role of vitamin A in mammalian reproduction and embryonic development. Annu Rev Nutr 22: 347-381, 2002.

12. Okano J, Suzuki S and Shiota K: Involvement of apoptotic cell death and cell cycle perturbation in retinoic acid-induced cleft palate in mice. Toxicol Appl Pharmacol 221: 42-56, 2007.

13. Yu Z, Lin J, Xiao Y, Han J, Zhang X, Jia H, Tang Y and Li Y: Induction of cell-cycle arrest by all-trans retinoic acid in mouse embryonic palatal mesenchymal (MEPM) cells. Toxicol Sci 83: 349-354, 2005.

14. Mercader J, Granados N, Bonet ML and Palou A: All-trans retinoic acid decreases murine adipose retinol binding protein 4 production. Cell Physiol Biochem 22: 363-372, 2008.

15. Zhang Y, Dong S, Wang W, Wang J, Wang M, Chen M, Hou J and Huang H: Activation of Notch1 inhibits medial edge epithelium apoptosis in all-trans retinoic acid-induced cleft palate in mice. Biochem Biophys Res Commun 477: 322-328, 2016.

16. Ross SA, McCaffery PJ, Drager UC and De Luca LM: Retinoids in embryonal development. Physiol Rev 80: 1021-1054, 2000.

17. Wang M, Huang $\mathrm{H}$ and Chen $\mathrm{Y}: \mathrm{Smad} 2 / 3$ is involved in growth inhibition of mouse embryonic palate mesenchymal cells induced by all-trans retinoic acid. Birth Defects Res A Clin Mol Teratol 85: 780-790, 2009.

18. Abbott BD and Birnbaum LS: Retinoic acid-induced alterations in the expression of growth factors in embryonic mouse palatal shelves. Teratology 42: 597-610, 1990.

19. Ferguson MW: Palate development. Development 103 (Suppl): S41-S60, 1988

20. Campbell JL Jr, Smith MA, Fisher JW and Warren DA: Dose-response for retinoic acid-induced forelimb malformations and cleft palate: A comparison of computerized image analysis and visual inspection. Birth Defects Res B Dev Reprod Toxicol 71: 289-295, 2004.

21. Rocchi M, Covone A, Romeo G, Faraonio R and Colantuoni V: Regional mapping of RBP4 to 10q23-q24 and RBP1 to 3q21-q22 in man. Somat Cell Mol Genet 15: 185-190, 1989.

22. Blaner WS: Retinol-binding protein: The serum transport protein for vitamin A. Endocr Rev 10: 308-316, 1989.

23. Wolf G: Serum retinol-binding protein: A link between obesity, insulin resistance, and type 2 diabetes. Nutr Rev 65: 251-256, 2007. 
24. Vaisbuch E, Romero R, Mazaki-Tovi S, Erez O, Kim SK, Chaiworapongsa T, Gotsch F, Than NG, Dong Z, Pacora P, et al: Retinol binding protein 4-a novel association with early-onset preeclampsia. J Perinat Med 38: 129-139, 2010.

25. Singh AT, Evens AM, Anderson RJ, Beckstead JA, Sankar N, Sassano A, Bhalla S, Yang S, Platanias LC, Forte TM, et al: All trans retinoic acid nanodisks enhance retinoic acid receptor mediated apoptosis and cell cycle arrest in mantle cell lymphoma. Br J Haematol 150: 158-169, 2010.

26. Radu M, Soprano DR and Soprano KJ: S10 phosphorylation of p27 mediates atRA induced growth arrest in ovarian carcinoma cell lines. J Cell Physiol 217: 558-568, 2008.

27. Zhang ML, Tao Y, Zhou WQ, Ma PC, Cao YP, He CD, Wei J and Li LJ: All-trans retinoic acid induces cell-cycle arrest in human cutaneous squamous carcinoma cells by inhibiting the mitogen-activated protein kinase-activated protein 1 pathway. Clin Exp Dermatol 39: 354-360, 2014.

28. Wesley UV, Hatcher JF and Dempsey RJ: Sphingomyelin synthase 1 regulates Neuro-2a cell proliferation and cell cycle progression through modulation of p27 expression and Akt signaling. Mol Neurobiol 51: 1530-1541, 2015.

29. Fillies T, Woltering M, Brandt B, Van Diest JP, Werkmeister R, Joos $\mathrm{U}$ and Buerger $\mathrm{H}$ : Cell cycle regulating proteins $\mathrm{p} 21$ and $\mathrm{p} 27$ in prognosis of oral squamous cell carcinomas. Oncol Rep 17: $355-359,2007$.
30. Dubey RK, Fingerle J, Gillespie DG, MiZ, Rosselli M, Imthurn B and Jackson EK: Adenosine attenuates human coronary artery smooth muscle cell proliferation by inhibiting multiple signaling pathways that converge on Cyclin D. Hypertension 66: 1207-1219, 2015.

31. Han YH, Gao B, Huang JH, Wang Z, Guo Z, Jie Q, Yang L and Luo ZJ: Expression of CD147, PCNA, VEGF, MMPs and their clinical significance in the giant cell tumor of bones. Int J Clin Exp Pathol 8: 8446-8452, 2015.

32. Shen H, Zhou E, Wei X, Fu Z, Niu C, Li Y, Pan B, Mathew AV, Wang X, Pennathur S, et al: High density lipoprotein promotes proliferation of adipose-derived stem cells via S1P1 receptor and Akt, ERK1/2 signal pathways. Stem Cell Res Ther 6: 95, 2015.

33. Zhang XJ and Jia SS: Fisetin inhibits laryngeal carcinoma through regulation of $A K T / N F-\kappa B / m T O R$ and ERK1/2 signaling pathways. Biomed Pharmacother 83: 1164-1174, 2016.

34. Li F, Xia K, Sheikh SA, Cheng J, Li C and Yang T: Involvement of RBP4 in hyperinsulinism-induced vascular smooth muscle cell proliferation. Endocrine 48: 472-482, 2015.

35. Li F, Xia K, Sheikh MS, Cheng J, Li C and Yang T: Retinol binding protein 4 promotes hyperinsulinism-induced proliferation of rat aortic smooth muscle cells. Mol Med Rep 9: 1634-1640, 2014.

36. Takebayashi K, Sohma R, Aso Y and Inukai T: Effects of retinol binding protein- 4 on vascular endothelial cells. Biochem Biophys Res Commun 408: 58-64, 2011. 